\title{
Process control performance evaluation in the case of variable set-point with experimental applications
}

\author{
Riccardo Bacci di Capaci ${ }^{\mathrm{a}, \mathrm{b}}$, Claudio Scali ${ }^{\mathrm{a}}$ \\ ${ }^{a}$ Department of Civil and Industrial Engineering, University of Pisa, Pisa, Italy \\ ${ }^{b}$ e-mail: riccardo.bacci@ing.unipi.it
}

\begin{abstract}
Purpose of this paper is to contribute with some refinements to recent methods of analysis of control loop performance, based on the well-established principle of Internal Model Control (IMC). Lower limits for the absolute value of the integral of control error (IAE) and the total variation of control action (TV) are assumed as reference values for a control considered good or at least acceptable. The overall performance index assumes as benchmark a controller tuned according to rules of S(implified)IMC technique and is appropriately defined with respect to the lower limits of the two metrics IAE and TV. This allows the assessment of control loop performance, that is, the validity of tuning for PID-type controllers in response to different types of reference change. In fact, one can assess performance in the case of set-point changes as steps, ramps, or generic varying trends over time. In order to demonstrate the validity of the refined technique, several examples of simulation, case studies on a pilot plant, and real industrial data are presented.
\end{abstract}

Keywords: Process control; loop performance monitoring and assessment; PID controller tuning; IMC; SIMC

\section{Introduction}

Set-point changes are pretty common in the process industry and may occur due to various scenarios. Inner control loops in cascade configurations are typically subject to continuous setpoint variations, due to corrections imposed by the master control loop in order to track the external reference or to reject oscillating external disturbances. Also the control loops under advanced structures, as model predictive control (MPC) and real time optimization (RTO), operate with variable references, and usually show small fluctuations, nonstationary time trends and slow drifts. In addition, control room operators or automatic preset programs may impose desired trajectories to references in loops operating in batch mode or subject to frequent variations of steady-states conditions.

For example, nowadays, variable operating conditions are typical scenarios of power plants. The reason lays in the fact that high seasonality and variability of electricity production from renewable sources has enormous impact on both production and distribution networks. ${ }^{[1]}$ Therefore, among the many aspects, several traditional power plants, especially, combined cycle plants, but also coal-fired plants, which were originally designed for base load applications and steady-state conditions, now operate on a more flexible basis, that is, with an intermittent program or with variable set-points due to fluctuations of energy load which they are requested to deliver. [2] 3]

Generally speaking, monitoring and assessment of performance of control systems of industrial plants are important topics in process control. The deterioration in performance is, in fact, a fairly common phenomenon and manifests with sluggish or oscillating trends of control variables. Oscillations in control loops can cause many problems which affect normal operation of process plants. Typically, fluctuations increase variability of product quality, accelerate wear of equipment, move operating conditions away from optimality, and cause excessive or unnecessary consumption of energy and raw materials. 44 5]

It is well-known that the design of the closed-loop control system should simultaneously guarantee good ability to reject load disturbances and track set-point changes, and also allow robustness to variation of process characteristics, in order to preserve the trade-off among all these aspects. ${ }^{6]}$ Nevertheless, it has to be noted that specific methods for assessment of control system performance during transient and/or cyclic phases, as general-type reference changes, two-shift regimes, but also operations of start-up and shutdown, are rather scarce in the literature and hence highly desirable.

In this paper, the focus is expressly to performance assessment of control loops for set-point tracking, also known as servo-control mode. Revisions and refinements of two recent techniques ${ }^{[7]}$ for the analysis of performance of basic control loops when process is subject to changes of operating conditions are proposed. Lower limits for the absolute value of the integral of control error (IAE) and the total variation of control action (TV) are assumed as reference values for a control considered good or at least acceptable. The overall performance index assumes as benchmark a controller tuned according to rules of SIMC technique and is appropriately defined with respect to the lower limits of the two metrics, IAE and TV.

Novel aspects of the present paper can be considered the following. Firstly, the definition of a lower limit for TV of control action and for IAE of control error, in the case of variation of set-point as a series of ramps; secondly, the choice of SIMC 


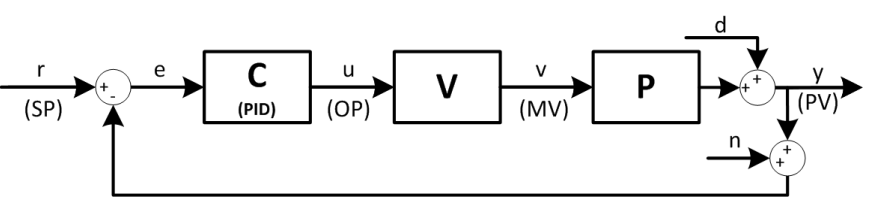

Figure 1: Diagram of a feedback control loop (SISO type).

technique as the reference for controller tuning. In addition, the effect of different tuning settings for the reference response on controller assessment results are shown. Cases study with different types of set-point variations, and data of different nature, as numerical examples, tests on a pilot plant, and industrial processes will be presented.

The remainder of the paper is organized as follows. The following section 2 describes the revised method, by briefly recalling the IMC and SIMC tuning rules. It also introduces the lower limit of IAE and TV indices, based on the IMC principle, by setting out some criteria to select an appropriate value for the tuning parameter $\left(\tau_{c}\right)$ in the reference response, and by presenting the index of loop performance. Then, sections 3, 4 and 5 provide numerical examples, experimental tests, and industrial data, respectively, in order to validate the reference values of indices, and illustrate the effectiveness of the performance index. The concluding remarks are given in section 6

\section{The refined approach}

In this paper, a SISO feedback control loop is considered, with $P(s), C(s), V(s)$ as the transfer functions of the process, PID-type controller, and control valve, respectively. The signals $r(t), u(t), v(t)$, and $y(t)$ are the reference (set-point, SP), the control action (OP), the valve position (MV), and the controlled variable (PV), respectively. In addition, $d(t)$ and $n(t)$ are the sequences of external disturbance and white Gaussian noise, respectively.

Note that MV signal is not usually available in traditional industrial plants, and, for a healthy control valve, input and output signals are basically equal: $u(t) \simeq v(t)$, and $V(s) \simeq 1$, or a very fast linear dynamics is present. On the opposite, a nonlinear dynamics in the valve, as for the presence of static friction (stiction), makes the two signals significantly different. [9]

\subsection{SIMC tuning rules}

Tuning rules of PID controllers based on the IMC method are now widely adopted in the industrial practice. ${ }^{[10]}$ In this work, the actual performance of controller is compared with the one achievable by a PI(D) controller tuned with the SIMC - Simplified IMC - rules. In particular, if the performance index is next to its maximum, then the current behavior of the control loop is next to the "ideal" behavior, obtainable with a SIMC-based controller.

The SIMC method employs specific tuning rules which allow a good balance between three conflicting factors in a control loop: that is, ability of tracking reference, limited control effort, and robust stability in closed-loop. ${ }^{[11]}$ The reference tracking ability is commonly evaluated by the Integral of Absolute Error (IAE):

$$
I A E=\int_{t=0}^{\infty}|e(t)| d t=\sum_{i=1}^{N}\left|S P_{i}-P V_{i}\right| T_{S}
$$

where $e(t)=S P-P V$ is the control error. Then, the control effort is usually quantified by the Total Variation (TV) of the control action:

$$
T V=\sum_{i=1}^{N-1}\left|O P_{i+1}-O P_{i}\right|
$$

where $O P$ is the control action, i.e., the controller output. Finally, the robust stability can be assessed in the frequency domain, by means of the peak of sensitivity function:

$$
M_{S}=\max _{\omega}\left|\frac{1}{1+P(j \omega) C(j \omega)}\right|
$$

where $P(j \omega)$ and $C(j \omega)$ represent the harmonic function of process and of PID-type controller, respectively. The PID controller $C(s)$ is here considered in the ideal (parallel, "noninteractive") formulation:

$$
C(s)=K_{c}\left(1+\frac{1}{T_{i} s}+T_{d} s\right)
$$

For simplicity, the process $P(s)$ is assumed linear time invariant (LTI); it is also stable, free of integrators and negative zeros. Its transfer function is therefore of the type:

$$
P(s)=K \frac{\prod_{j=1}^{M}\left(\alpha_{o, j} s-1\right)}{\prod_{i=1}^{N}\left(T_{o, i} s+1\right)} e^{-\theta_{o} s}
$$

where $\alpha_{o, j}, T_{o, i}$ are the time constants associated with zeros and poles, and $\theta_{o}$ is the time-delay. Consequently, a model of firstorder plus time-delay (FOPTD) can well approximate the process dynamics for practical purpose:

$$
P(s) \approx \frac{K}{\tau s+1} e^{-\theta s}
$$

where $K, \tau$, and $\theta$ are gain, time constant, and time-delay, respectively. Alternatively, a model of second-order plus timedelay (SOPTD) can be adopted:

$$
P(s) \approx \frac{K}{\tau_{1} s^{2}+\tau_{2} s+1} e^{-\theta s}
$$

where $\tau_{1}$ and $\tau_{2}$ are coefficients related with the two time constants.

The process model can be identified with a linear leastsquare regression and one of the many methods for time-delay estimation, 12$]$ on the basis of routine data collected in closedloop operation and without introducing extra experiments. Note that, being critical to the overall performance index, $\theta$ is the time-delay of the reduced-order model, and not the time-delay $\theta_{o}$ of the actual process that is approximated. To this end, the positive zeros $\left(1 / \alpha_{0, j}\right)$ of the actual process can be incorporated within the reduced-order model delay. 
The SIMC tuning rules ${ }^{[11]}$ provides the following parameters of PI-type controller to be used for a FOPTD model:

$$
K_{c}=\frac{\tau}{K\left(\tau_{c}+\theta\right)}, \quad T_{i}=\min \left\{\tau, 4\left(\tau_{c}+\theta\right)\right\}, \quad T_{d}=0
$$

where $\tau_{c}$ is the actual tuning parameter, in the sense that the smaller is its value, the faster is the time constant of the closedloop response. While the following parameters of PID-type controller are to be used for a SOPTD model:

$$
K_{c}=\frac{\tau_{2}}{K\left(\tau_{c}+\theta\right)}, \quad T_{i}=\min \left\{\tau_{2}, 4\left(\tau_{c}+\theta\right)\right\}, \quad T_{d}=\frac{\tau_{1}}{\tau_{2}}
$$

Note that by using SIMC tuning rules, the integral action is corrected with respect to the original IMC method, since for lag dominant processes $(\tau \gg \theta)$, e.g., integrating processes, the choice $T_{i}=\tau$ would result in a long settling time in response to input ("load") disturbances. [11]

In general, through IMC approaches, a direct synthesis of the controller is performed, and the following - desired - response in closed-loop to a set-point variation is imposed:

$$
\begin{aligned}
G_{r_{C L}}(s) & =\frac{Y(s)}{R(s)}=\frac{P(s) C(s)}{1+P(s) C(s)}=\frac{1}{\left(\tau_{c}+\theta\right) s+e^{-\theta s}} e^{-\theta s} \\
& \approx \frac{1}{\left(\tau_{c}+\theta\right) s+1-\theta s} e^{-\theta s}=\frac{1}{\tau_{c} s+1} e^{-\theta s}
\end{aligned}
$$

where $Y(s)$ and $R(s)$ are the Laplace transforms of $y(t)$ and $r(t)$, and first-order Taylor expansion is used to approximate the delay in denominator of $G_{r_{C L}}(s)$, namely: $e^{-\theta s} \approx 1-\theta$. Therefore, the desired closed-loop response is analogous to the openloop dynamics of a process of FOPTD type.

Note that $\tau_{c}$, the desired time constant of closed-loop response, becomes the actual tuning parameter, which has to be chosen by the user. As a rule, $\tau_{c}$ should be greater than the process time-delay $\theta$, since usually the response will be otherwise too aggressive or even oscillating, but not greater than the time constant in open-loop $\tau_{O L}$. In the case of SIMC tuning, i1 it is recommended to set the closed-loop time constant just equal to time-delay: $\tau_{c}=\theta$. In general, an appropriate value for $\tau_{c}$ can be selected by balancing the three first mentioned opposing factors: a quick response in closed-loop, a limited change in control action, and a high robust stability in closed-loop.

Finally, it has to be recalled that original IMC approach depends on the specific input, since the optimal algorithm of IMC controller is a complex function which also involves the minimum-phase part of the input. Therefore, the input type must be known in advance. This facts may also be relevant for a PID controller derived from IMC, implying that a controller may not be optimal for every input type. Following indications given by $\mathrm{Yu}$ and coworkers, ${ }^{[8]}$ an appropriate value for $\tau_{c}$ could be actually obtained on the basis of the reference variation. For example, in the case of ramp, $\tau_{c}$ is a function of the slope $k$ and the amplitude $A$. In this work, the reference controller $\hat{C}_{r}(s)$ is a pure PI for a FOPTD model, and a pure PID for a SOPTD process model, with suitable choices of $\tau_{c}$.

\subsection{Lower limits for IAE and TV}

In works of Yu et al., ${ }^{[7]}$ the lower limit for IAE index $\left(I A E_{0}\right)$ and for TV index $\left(T V_{0}\right)$ have been derived by using a IMCbased controller, for closed-loop response subject to a reference change as step and ramp, and also the lower limit for IAE index for a general-type reference has been established. Here below only some aspects of such studies are recalled. In the present work, this approach is refined, by using SIMC - instead of IMC - as reference for controller tuning, and the definition of the lower limit for TV in the case of general-type reference, and lower limit for IAE and TV, in the specific case of variation as a series of macroscopic ramps are introduced.

Step. In the work of Yu et al. ${ }^{[7}$, the lower limit of index IAE for step response has been expressed as:

$$
I A E_{0, \text { step }}=\Delta_{S P}\left(\tau_{c}+\theta\right)
$$

where the reference signal $r(t)$ undergoes a step change, for simplicity assumed from 0 to $\Delta_{S P}$, which is a positive real number. According to SIMC tuning rules, ${ }^{[1]}$ since $\tau_{c}=\theta$, the lower limit results:

$$
I A E_{0}=2 \theta \Delta_{S P}
$$

This expression has already been proposed in other works, 13 14] and is consistent with corresponding values obtained by Swanda and Seborg through simulations: $I A E_{0} \in\left[1.9 \theta \Delta_{S P}, 2.1 \theta \Delta_{S P}\right] .[15]$ The lower limit of TV index for the step has been instead obtained as a special case of the ramp. $\underline{8}$

Ramp. It is to be pointed out that lower limits in the case of ramp and for general types of set-point changes have not been studied in the literature with the same attention of step change. Moreover, in industrial practice, in particular in power plants, reference changes of control loops - and thus of operating conditions of process - are mostly obtained with gradual programs of ramps, rather than with sudden step changes. In addition, in many cases, values of ramp parameters are known a-priori. The load demand as set-point is typically received from a powergrid control center, so that the new desired value $A=k T$ is known in advanced, while the variation rate (the slope) $k$ and the time duration $T$ for the ramp are preset parameters by operators. As shown in Figure 2, the equation of ramp is:

$$
r(t)= \begin{cases}k t+r_{0} & \text { for } \quad 0 \leq t<T \\ k T+r_{0}=A+r_{0} & \text { for } \quad T \leq t<\infty\end{cases}
$$

where $r_{0}$ is the initial steady-state value. As shown by Yu et al., ${ }^{[7]}$ the lower limit of index IAE for the response to a ramp is:

$$
\begin{array}{lll}
I A E_{0, \theta \leq T}=k T\left(\tau_{c}+\theta\right) & \text { if } & \theta \leq T \\
I A E_{0, \theta>T}=k T\left(\tau_{c}+\theta\right) & \text { if } & \theta>T
\end{array}
$$

If the ramp has a negative slope $(k<0)$, one gets: $I A E_{0}=$ $-k T\left(\tau_{c}+\theta\right)$. Therefore, in general, the lower limit of index IAE is simply:

$$
I A E_{0, \text { ramp }}=|k T|\left(\tau_{c}+\theta\right)=|A|\left(\tau_{c}+\theta\right)
$$




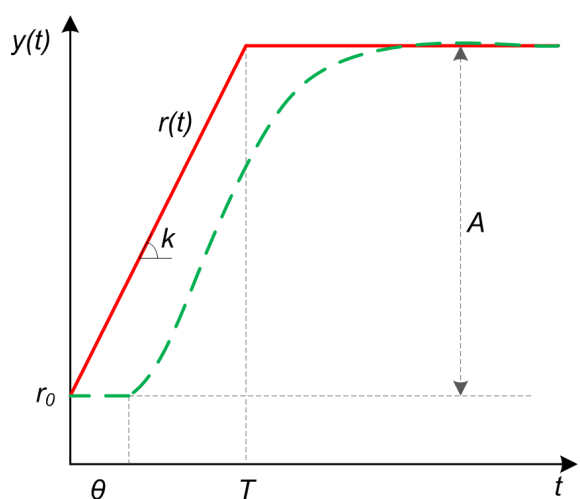

Figure 2: Reference change as a ramp signal.

and this applies regardless the relative values of $\theta$ and $T$.

Equation 15 shows that the lower limit of index IAE for a closed-loop response to a ramp does not depend on the slope $k$, and it is actually the same as what obtained for the corresponding step response; compare (11), where $\Delta_{S P}=A .{ }^{[7]}$ This is explained by noting that: (i) the step change is a special case of ramp with a infinite slope: $k \rightarrow \infty$ in 13, (ii) the response to a ramp with a smaller slope requires a higher settling time with respect to the step response of equal overall amplitude, despite the fact that for each time interval, the error in response to the ramp is smaller.

The lower limit for TV index has been demonstrated as: $[8]$

$T V_{0, \mathrm{ramp}}= \begin{cases}\left|\frac{k}{K}\right|\left(\frac{A}{k}+2\left(\tau-\tau_{c}\right)\left(1-e^{-A / k \tau_{c}}\right)\right) & \text { for } \quad 0<\tau_{c} \leq \tau \\ \left|\frac{A}{K}\right| & \text { for } \quad \tau<\tau_{c}<\infty\end{cases}$

where $K$ is the gain, and $\tau$ is the time constant of FOPTD model. Note that for a SOPTD process model the lower limit of TV would be much more complex, by involving the two parameters $\tau_{1}$ and $\tau_{2}$. In the case of step change, since $k \rightarrow \infty$, the lower limit of TV is obtained by applying the concept of infinitesimal equivalent to $[16)$, that is:

$$
T V_{0, \text { step }}=\lim _{k \rightarrow \infty} T V_{0}= \begin{cases}\left|\frac{A}{K}\right| \frac{2 \tau-\tau_{c}}{\tau_{c}} & \text { for } \quad 0<\tau_{c} \leq \tau \\ \left|\frac{A}{K}\right| & \text { for } \quad \tau<\tau_{c}<\infty\end{cases}
$$

Generic variation. Whether the reference signal $r(t)$ varies from a generic value to another and overall follows a generic path, a trend similar to one shown in Figure 3 is obtained. A series of small ramp signals can approximate the original setpoint: 7

$$
r(t) \approx r_{1}(t)+r_{2}(t)+\ldots+r_{N}(t)
$$

where:

$$
r_{i}(t)= \begin{cases}r_{i-1} & 0 \leq t<t_{i-1} \\ k_{i}\left(t-t_{i-1}\right)+r_{i-1} & t_{i-1} \leq t<t_{i} \\ k_{i}\left(t_{i}-t_{i-1}\right)+r_{i-1}=A_{i}+r_{i-1} & t_{i} \leq t<\infty\end{cases}
$$

where $k_{i}$ is the slope of generic ramp $r_{i}(t)$; while $r_{0}$ and $r_{N}$ are the initial and final steady-state values for $r(t)$; that is, $r(t)=$

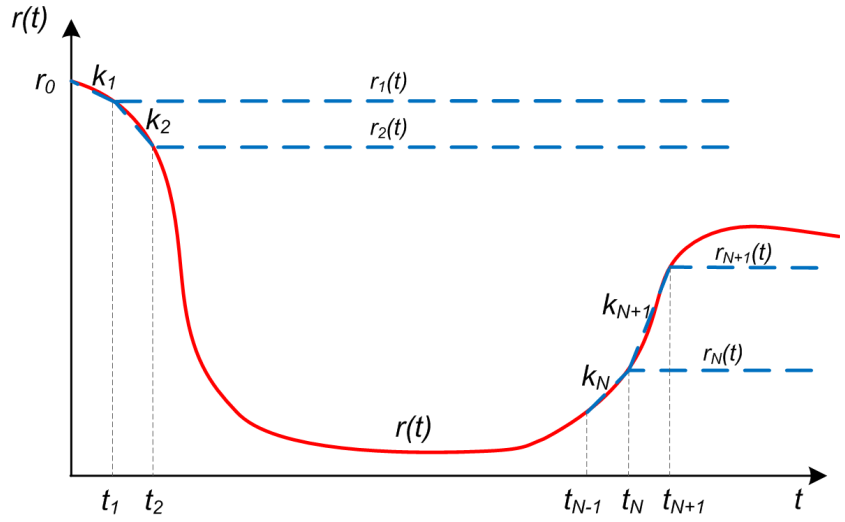

Figure 3: Generic variable set-point and decomposition in sequence of ramps.

$r\left(t_{0}\right)=r_{0}$ for $t \leq t_{0}$, and $r(t)=r\left(t_{N}\right)=r_{N}$ for $t \geq t_{N}$. Note that at the limit, the difference $t_{i}-t_{i-1}$ equals the sampling period $T_{S}$, and $N$ becomes the number of samples from $t_{0}$ to $t_{N}$. From (15), applying the superposition principle, since the system is assumed LTI, one can get the lower limit of IAE in the generic case as:

$$
\begin{aligned}
I A E_{0} & \approx I A E_{0}\left[r_{1}(t)+\ldots+r_{N}(t)\right] \\
& \leq I A E_{0}\left[r_{1}(t)\right]+\ldots+I A E_{0}\left[r_{N}(t)\right] \\
& =\left(\left|k\left(t_{1}-t_{0}\right)\right|+\ldots+\left|k\left(t_{N}-t_{N-1}\right)\right|\right)\left(\theta+\tau_{c}\right)
\end{aligned}
$$

where $I A E_{0}\left[r_{i}(t)\right]$ is the lower limit of index IAE for the $i$-th ramp signal $r_{i}(t)$.

Equality in 20 is under two conditions: (i) all slopes $r_{i}(t)$ $(i=1, \ldots, N)$ have the same sign, i.e., the set-point is monotone increasing or decreasing; (ii) in the case of change of sign between $r_{i}(t)$ and $r_{i+1}(t)$, that is, if the set-point changes direction, then $r_{i}(t)$ has to be constant and equal to the value $r\left(t_{i}\right)$ for a time sufficient to the control loop to reach the new steady-state. Under these two conditions, by ignoring approximation errors in (18), the lower limit of the index IAE becomes simply: ${ }^{[7]}$

$$
I A E_{0, \text { gen }}=\sum_{i=1}^{N}\left|r_{i}-r_{i-1}\right|\left(\tau_{c}+\theta\right)=\sum_{i=1}^{N}\left|A_{i}\right|\left(\tau_{c}+\theta\right)
$$

Therefore, the response to step (11) and ramp (14) are two special cases of the general 21.

Similarly, in the present work it is suggested to express the control signal $u(t)$, corresponding to the reference $r(t)$, as a set of small contributions:

$$
u(t) \approx u_{1}(t)+u_{2}(t)+\ldots+u_{N}(t)
$$

As regards the lower limit of index TV, starting from (16) and applying the superposition principle, one gets:

$$
\begin{aligned}
T V_{0, \text { gen }} & \approx T V_{0}\left[u_{1}(t)+\ldots+u_{N}(t)\right] \\
& \leq T V_{0}\left[u_{1}(t)\right]+\ldots+T V_{0}\left[u_{N}(t)\right] \\
& =\sum_{i=1}^{N} T V_{0}\left[u_{i}(t)\right]
\end{aligned}
$$


where $T V_{0}\left[u_{i}(t)\right]$ is the lower limit for index TV of $i$-th control action $u_{i}(t)$ associated with the $i$-th $\operatorname{ramp} r_{i}(t)$, expressed in a similar way to $[16)$ :

$$
T V_{0}\left[u_{i}(t)\right]= \begin{cases}\left|\frac{k_{i}}{K}\right|\left(\frac{A_{i}}{k_{i}}+2\left(\tau-\tau_{c}\right)\left(1-e^{-A_{i} / k_{i} \tau_{c}}\right)\right) & \text { for } 0<\tau_{c} \leq \tau \\ \left|\frac{A_{i}}{K}\right| & \text { for } \tau<\tau_{c}<\infty\end{cases}
$$

where $A_{i}$ and $k_{i}$ are the net amplitude and the slope of the $i$-th ramp signal $r_{i}(t)$. The equality in (23) applies under conditions similar to 20. But note that condition (ii) is even less feasible when is applied to the control action, and approximation errors on single contributions $T V_{0}\left[u_{i}(t)\right]$ are rather high.

Therefore, it is worth noticing that it is hard to obtain a completely reliable analytical expression for the lower limit of TV in the case of generic variation of set-point, especially whether $r(t)$ varies very quickly, as for the case of internal controller in a cascade configuration, when the master loop which moves the reference is tightly tuned. Anyway, smaller errors are awaited when reference trajectory is slow-varying, as for the case of control loops under advanced configurations.

Series of ramps. A particular case, but actually quite common in industrial practice, is the one represented by a reference signal comprised of a pure series of $M$ macroscopic ramps of different slope and amplitude. The case in which $M \ll N$ is here considered, that is, when individual ramps do not approach infinitesimal contributions $r_{i}(t)$ as for the case of (19). In this scenario, a generalization of (16) can be employed by committing a very small approximation. The analytical expression of the lower limit of TV as the sum of contribution of individual ramps becomes:

$$
\begin{aligned}
& T V_{0, \mathrm{sr}} \approx \sum_{i=1}^{N} T V_{0}\left[u_{i}(t)\right] \approx \sum_{m=1}^{M} T V_{0}\left[u_{m}(t)\right]= \\
& = \begin{cases}\sum_{m=1}^{M}\left|\frac{k_{m}}{K}\right|\left(\frac{A_{m}}{k_{m}}+2\left(\tau-\tau_{c}\right)\left(1-e^{-A_{m} / k_{m} \tau_{c}}\right)\right) & \text { for } 0<\tau_{c} \leq \tau \\
\sum_{m=1}^{M}\left|\frac{A_{m}}{K}\right| & \text { for } \tau<\tau_{c}<\infty\end{cases}
\end{aligned}
$$

where $A_{m}$ and $k_{m}$ are the net amplitude and the slope of the $m$-th ramp signal $r_{m}(t)$. Analogously, from (14) with little approximation, the expression of the lower limit of IAE in the case of a series of $M$ ramps becomes as following:

$$
I A E_{0, \mathrm{sr}}=\sum_{m=1}^{M}\left|k_{m} T_{m}\right|\left(\tau_{c}+\theta\right)=\sum_{m=1}^{M}\left|A_{m}\right|\left(\tau_{c}+\theta\right)
$$

Note that parameter $M$, that is, the number of macroscopic ramps approximating the general-type reference $r(t)$, can be defined by the user or set automatically. Similarly, the start and final points of each linear segment of trajectory can be assigned manually or automatically. An example of manual procedure is the following. Once a significant data-set is registered, the reference trajectory is divided into a first set of data windows, which typically correspond to the segments with monotonic trend, detected by a visual inspection. Then, each major window can be further divided in smaller windows in order to get a suitable series of $M$ ramps, by connecting the start and final points of each window. Alternative, an automatic procedure can be employed, by simply dividing the general-type trajectory in $M$ segments of constant length or - better - by detecting local stationary points. In this last case, the task can be rather complicated for noisy signals, since a robust algorithm for the identification of local peaks, valleys, and inflection points is required. This may mean fitting the original signal with spline functions, and use moving-average methods and/or cluster techniques.

Data-driven and model-based approach. In general terms, as alternative to the previous analytic formulas for $I A E_{0}$ and $T V_{0}$ 11 26, is here also proposed to compute lower limits of performance indices by using values of $I A E$ and $T V$ from operating data, via (1) and 2. This means considering explicitly as reference performance the one that would be obtained by controlling the identified process model $\hat{P}(s)$ with a hypothetical controller $\hat{C}_{r}(s)$ tuned according to SIMC rules.

In order to reduce the effect of noise, instead of actual indices $\left(I A E_{A c t}, T V_{A c t}\right)$ from registered data, one can consider the values obtained with the estimate of controlled variable $\hat{y}(t)$ and control action $\hat{u}(t)$, on the basis of the identified process model $\hat{P}(s)$ and the transfer functions in closed-loop mode:

$$
\hat{Y}(s)=\frac{\hat{P}(s) C(s)}{1+\hat{P}(s) C(s)} R(s), \quad \hat{U}(s)=\frac{C(s)}{1+\hat{P}(s) C(s)} R(s)
$$

which yield the re-simulated signals $(\hat{y}(t), \hat{u}(t))$, that are totally noise-free. Analogously, estimated references for time trends of controlled variable $\hat{y}_{r}(t)$ and control action $\hat{u}_{r}(t)$ are obtained from:

$$
\hat{Y}_{r}(s)=\frac{\hat{P}(s) \hat{C}_{r}(s)}{1+\hat{P}(s) \hat{C}_{r}(s)} R(s), \quad \hat{U}_{r}(s)=\frac{\hat{C}_{r}(s)}{1+\hat{P}(s) \hat{C}_{r}(s)} R(s)
$$

Obviously, this model-based approach requires, besides to a reliable process identification, also a safe description of the whole feedback control loop, which might not always feasible in practical applications.

\subsection{The performance indices}

As said before, the lower limits $\left(I A E_{0}\right.$ and $\left.T V_{0}\right)$ are taken as reference values for the corresponding performance index. Two dimensionless ratios are firstly used $\left(\eta_{I A E}, \eta_{T V}\right)$, and then a global index $(\eta)$ is computed:

$$
\begin{gathered}
\eta_{I A E}=\frac{\min \left(I A E_{0}, I A E_{A c t}\right)}{\max \left(I A E_{0}, I A E_{A c t}\right)} \\
\eta_{T V}=\frac{\min \left(T V_{0}, T V_{A c t}\right)}{\max \left(T V_{0}, T V_{A c t}\right)} \\
\eta=\eta_{I A E} \cdot \eta_{T V}
\end{gathered}
$$

where $I A E_{A c t}$ is the (actual) integral index of the absolute error, as in (1). Similarly, $T V_{A c t}$ is the (actual) total variation of the control action, as in 2). Both performance indices assume values in the interval $(0,1]$, with ideal value equal to 1 . Combining the two indices $\left(\eta_{I A E}, \eta_{T V}\right)$, the global performance index $(\eta)$ is defined, which also lies in $(0,1]$, with 1 as ideal value. $[8]$ 
In details, if $\eta \rightarrow 1$, the actual performance is regarded as satisfactory, since the actual controller tends to the reference one, tuned with rules of SIMC technique. In particular, both $I A E_{A c t}$ and $T V_{A c t}$ are close to reference values: $I A E_{0}$ and $T V_{0}$. On the opposite, if $\eta \rightarrow 0$, the actual performance is far from what obtained by a PID controller tuned with SIMC rules; i.e., at least one index between $I A E_{A c t}$ and $T V_{A c t}$ is away from reference value.

It must be observed that index $\eta$, being based on IAE and $\mathrm{TV}$, considers simultaneously the closed-loop response and the control action. Note that the actual integral of absolute error could be even lower than the reference value: $I A E_{A c t}<I A E_{0}$. However, this situation is not to be preferred, since actual controller could have lower robust stability and it could cause unacceptable variations in the control action. In addition, note that $\eta$ provides a measure of control loop performance in the case of set-point tracking, regardless the tuning rule followed by the actual controller, and it is also applicable to most common schemes of industrial control. 8

As outlined before, as important limitations of the method, the process must be linear and time-invariant (LTI), and loop instruments (sensors, actuators, valves) must work properly. This implies that the eventual source of malfunction has to be of linear type, due to an internal source (controller tuning) or at the limit an external source (process disturbances). As general note, this method of performance assessment should be applied only when the presence of nonlinearities is excluded. For this purpose, referring to scheme of Figure 1, well-established techniques for detection of static friction in control valves could be used. [9] In particular, the recent method of Dambros et al., [16] especially suited for loops with variable reference signal, could be employed as a preliminary test of the analysis.

\subsection{Phases of the analysis}

Under the assumptions and constraints previously cited, phases of methodology for loop assessment are:

1. Collect routine data of input $u(t)$ and output $y(t)$, estimate process dynamics with a model $\hat{P}(s)$ of first or second order plus time-delay, and then evaluate key parameters: i.e., for FOPTD, $\hat{K}, \hat{\tau}$, and $\hat{\theta}$. On the basis of set-point $r(t)$ and controller transfer function $C(s)$, estimate the closed-loop response as $\hat{y}(t)$. If $\hat{y}(t)$ well captures dynamics of real data $y(t)$ - typically if a fitting index is sufficiently high - go to step 2; otherwise choose another data set, and repeat step 1. The following fitting index on the controlled variable $(y$, that is, $P V)$ is employed in this work:

$$
F_{P V}=100 \cdot\left(1-\frac{\|y(t)-\hat{y}(t)\|}{\|y(t)-\bar{y}(t)\|}\right)
$$

where $\bar{y}(t)$ is the average value of $P V$, the operator $\|\cdot\|$ denotes the Euclidean norm, and a good process identification is considered for $F_{P V} \geq 80 \%$.

2. Choose the time constant $\tau_{c}$ of the reference response in closed-loop. A good value can be the estimate of process time-delay: $\tau_{c}=\hat{\theta}$, as suggested in SIMC method. [11] Alternatively, the minimum value between current time constant, identified in open-loop and closed-loop, can be used: $\tau_{c}=\min \left\{\hat{\tau}_{O L}, \hat{\tau}_{C L}\right\}$. Note that the time constant in closedloop $\hat{\tau}_{C L}$ is estimated on the basis of set-point $r(t)$ and controlled variable $y(t)$ by employing another FOPTD model, as in (10).

3. Compute $I A E_{A c t}$ as in (1), estimate the reference value $I A E_{0}$ according to 21) or 26), evaluate the performance index $\eta_{I A E}$. Then, compute $T V_{A c t}$ as in (2), estimate the reference value $T V_{0}$ according to (24) or 25, and compute the performance index $\eta_{T V}$.

4. Finally, compute the global index $\eta$. If $\eta$ is large enough, typically if $\eta \geq 0.8$, then the control loop has acceptable performance. Otherwise, the performance is considered poor and corrective actions, as controller retuning or adoption of different schemes, are recommended. Obviously, in the case of retuning, the reference parameters of SIMCbased controller are suggested, as in (8) and 9].

In the following sections, cases study with different types of set-point variations, and data of different nature are presented. Process parameters are identified with a FOPTD model, that is, a time-discrete linear Auto Regressive $\operatorname{ARX}(1,1)$ model, and with a grid-search for the time-delay $\theta$. 17]

\section{Simulation examples}

In order to demonstrate the effectiveness of the method, some simulation cases study are firstly presented. The process $P(s)$ has a linear dynamics described by a FOPTD model:

$$
P(s)=\frac{3}{100 s+1} e^{-7 s}
$$

The control valve has an ideal behavior, so that $v(t)=u(t) \forall t$. A white Gaussian noise signal $n(t)$, with zero-mean and variance equal to 0.1 , is introduced into the process variable, by producing a situation of noise signal ratio (NSR) equal around $20 \%$. The set-point program is of general-type and consists of a series of continuous variations, that is, a sequence of step, ramp, sinusoidal, exponential, and parabolic trends:

$$
r(t)= \begin{cases}20 & \text { for } 0 \leq t<25 \\ 10 & \text { for } 25 \leq t<250 \\ 0.8 t-210 & \text { for } 250 \leq t<275 \\ 30 & \text { for } 275 \leq t<475 \\ 10-7.5 \sin \left(\frac{\pi}{160}(t-475)\right) & \text { for } 475 \leq t<795 \\ 30 & \text { for } 795 \leq t<950 \\ 11-\exp \left(\frac{\pi}{50}(t-950)\right) & \text { for } 950 \leq t<1000 \\ 10.73 & \text { for } 1000 \leq t<1200 \\ -10.73+0.001(t-1200)^{2} & \text { for } 1200 \leq t<1355 \\ 33.3 & \text { for } 1355 \leq t<\infty\end{cases}
$$


Table 1: Tuning features and parameters.

\begin{tabular}{llll}
\hline Case \# & Tuning & $K_{c}$ & $T_{i}$ \\
\hline 1 & Aggressive & 3.5 & 60 \\
2 & Sluggish & 1.5 & 200 \\
3 & Good & 2.38 & 56 \\
4 & Fair & 2.74 & 103.5 \\
\hline
\end{tabular}

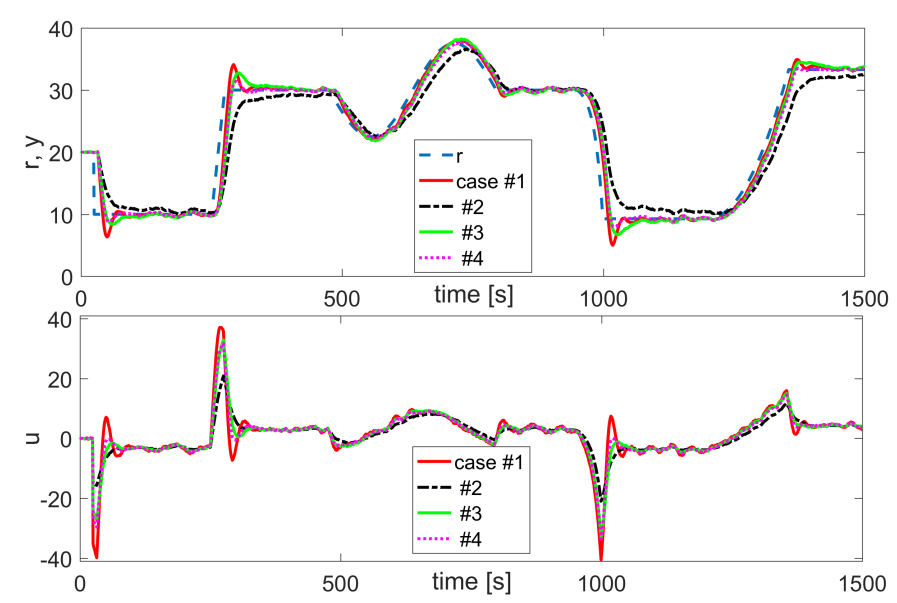

Figure 4: Time trends for the set-point changes; top) controlled variable; bottom) control action.

Four cases are analyzed, with different tuning parameters for the PI controller, as reported in Table 1 . The various time trends of output $y(t)$ in response to reference $r(t)$, and control action $u(t)$ are shown in Fig. 4. For each case, on the basis of registered signals $u(t)$ and $y(t)$, a FOPTD model $\hat{P}(s)$ is identified (see Table 2). Note that all models are - correctly - very consistent.

Results for the proposed approach. Firstly, in order to assess controller performance, the model-based approach is used. Therefore, two simulations are performed: i) with the actual controller, to get $\hat{y}(t)$ and $\hat{u}(t)$, and hence eliminate the effect of noise on numerical values of indices $\left(I A E_{A c t}, T V_{A c t}\right)$; ii) with the ideal controller $\hat{C}_{r}$, tuned with SIMC rules, to get $\hat{y}_{r}(t)$ and $\hat{u}_{r}(t)$ for the definition of references $\left(I A E_{0}, T V_{0}\right)$. For each case, as suggested by SIMC method, the estimate of process timedelay is imposed as the time constant of the reference response in closed-loop: $\tau_{c}=\hat{\theta}$.

Note that, since all identified process models are very consistent, reference controller $\hat{C}_{r}$ has always pretty the same parameters, that is, $K_{c}^{r} \simeq 2.38$ and $\tau_{I}^{r}=56$, according to (8). Then, performance index for IAE and TV are evaluated, and finally global index $\eta$ is computed. All the values are shown in Table 3 .

It can be observed that for case \#1, corresponding to an ag-

Table 2: Identified process dynamics as FOPTD models.

\begin{tabular}{|c|c|c|c|c|}
\hline & case \#1 & case \#2 & case \#3 & case \#4 \\
\hline$\hat{P}$ & $\frac{3.001}{100.01 s+1} e^{-7 s}$ & $\frac{2.998}{99.43 s+1} e^{-7 s}$ & $\frac{3.001}{99.82 s+1} e^{-7 s}$ & $\frac{3.001}{99.88 s+1} e^{-7 s}$ \\
\hline
\end{tabular}

Table 3: Results of loop performance assessment $\left(\tau_{c}=\hat{\theta}\right)$.

\begin{tabular}{|c|c|c|c|c|c|c|c|c|c|c|c|}
\hline $\begin{array}{l}\text { Case } \\
\#\end{array}$ & $\tau_{c}$ & $I A E_{0}$ & $I A E_{A c t}$ & $\eta_{I A E}$ & $T V_{0}$ & $T V_{A c t}$ & $\eta_{T V}$ & $\eta$ & $\begin{array}{l}F_{P V} \\
{[\%]}\end{array}$ & Verdict & \\
\hline 1 & 7 & 1741.2 & 1324.1 & 0.76 & 261.4 & 400.1 & 0.65 & 0.50 & 98.2 & Not Good & $\checkmark$ \\
\hline 2 & 7 & 1739.4 & 2985.3 & 0.58 & 260.1 & 160.5 & 0.62 & 0.36 & 98.0 & Not Good & $\checkmark$ \\
\hline 3 & 7 & 1740.6 & 1737.7 & 0.99 & 260.9 & 261.4 & 0.99 & 0.99 & 98.3 & (Very) Good & $\checkmark$ \\
\hline 4 & 7 & 1740.8 & 1423.4 & 0.82 & 261.1 & 275.8 & 0.95 & 0.77 & 98.3 & (Fairly) Good & $\checkmark$ \\
\hline
\end{tabular}

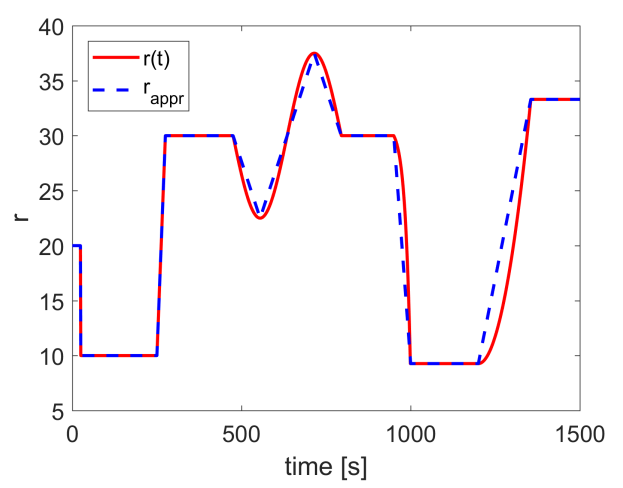

Figure 5: General-type reference trajectory $r(t)$ and sequence of $M=7$ ramps used as an approximation.

gressive tuning, a low performance index $\eta$ is obtained, due to an excessively variable control action $\left(T V_{A c t}>T V_{0}\right)$, which implies $\eta_{T V}=0.65$. Case \#2, expression of a sluggish tuning, is actually assessed with poor performance: $\eta=0.36$. In this case, both indices, $\eta_{I A E}$ and $\eta_{T V}$, are very low, being $I A E_{A c t}>I A E_{0}$ and $T V_{A c t}<T V_{0}$. On the contrary, case \#3, expression of an appropriate tuning, is particularly effective. In fact, actual performance tends to the ideal value $(\eta \rightarrow 1)$ since the controller is tuned just according to SIMC rules, which constitute the reference. The case \#4, when the controller follows

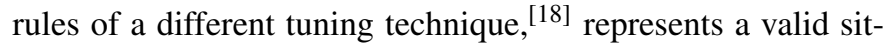
uation. The performance index is close to the threshold value ( $\eta \simeq 0.8$ ); that is, the controller has a fairly acceptable behavior, although considered inferior to the reference SIMC controller. Finally, note that fitting index $F_{P V}$ of the controlled variable is always very high, which gives an indication of correctness of process identification, and reliability of performance assessment.

Testing the approximation of general-type reference with a series of ramps. Then, controller performance is reevaluated in order to verify the validity of proposed relations for reference indices, $I A E_{0}$ (26) and $T V_{0}$ (25), specially designed for a pure series of ramps. To this aim, the general-type reference trajectory $r(t)$ (34) is approximated by using a series of $M=7$ ramps, as shown in Fig. 5 . Note that $A=[-10,20,-7.5,15,-7.5,-20.73,24.03]$ and $k=$ $[\infty, 0.8,-7.5 / 80,15 / 160,-7.5 / 160,-20.73 / 50,24.03 / 155]$ are vectors containing the net amplitudes and the slopes of the corresponding ramps, as defined in (13), obtained by linearizing each macroscopic variation between the starting and final point. Note that the sinusoidal variation has been approximated by three ramps, while the first step is considered as a ramp with infinite slope.

The new assessment results are shown in Table 4 Note that 
Table 4: Results of performance assessment when reference is approximated by a series of ramps.

\begin{tabular}{llllllll}
\hline Case \# & $I A E_{0}$ & $\eta_{I A E}$ & $T V_{0}$ & $\eta_{T V}$ & $\eta$ & Verdict & \\
\hline 1 & 1466.7 & 0.90 & 221.5 & 0.55 & 0.50 & Not Good & $\checkmark$ \\
2 & 1466.7 & 0.49 & 220.5 & 0.73 & 0.36 & Not Good & $\checkmark$ \\
3 & 1466.7 & 0.84 & 221.1 & 0.85 & 0.71 & (Fairly) Good & $\checkmark$ \\
4 & 1466.7 & 0.97 & 221.2 & 0.80 & 0.78 & (Fairly) Good & $\checkmark$ \\
\hline
\end{tabular}

Table 5: Results of performance assessment for different tuning settings of the reference response.

\begin{tabular}{|c|c|c|c|c|c|c|c|c|}
\hline \multicolumn{9}{|c|}{ Choice \#1: $\quad$ IMC with $\tau_{c}=\hat{\theta}$} \\
\hline Case \# & $\hat{\theta}$ & $I A E_{0}$ & $\eta_{I A E}$ & $T V_{0}$ & $\eta_{T V}$ & $\eta$ & \multicolumn{2}{|l|}{ Verdict } \\
\hline 1 & 7 & 1541.5 & 0.86 & 242.3 & 0.61 & 0.52 & Not Good & $\checkmark$ \\
\hline 2 & 7 & 1541.5 & 0.49 & 240.6 & 0.67 & 0.34 & Not Good & $\checkmark$ \\
\hline 3 & 7 & 1541.5 & 0.99 & 241.7 & 0.99 & 0.99 & (Very) Good & $\checkmark$ \\
\hline 4 & 7 & 1541.5 & 0.92 & 242.0 & 0.87 & 0.81 & Good & $\checkmark$ \\
\hline \multicolumn{9}{|c|}{ Choice \#2: $\quad$ IMC with $\tau_{c}=\min \left\{\hat{\tau}_{O L}, \hat{\tau}_{C L}\right\}$} \\
\hline Case \# & $\tau_{C L}$ & $I A E_{0}$ & $\eta_{I A E}$ & $T V_{0}$ & $\eta_{T V}$ & $\eta$ & \multicolumn{2}{|l|}{ Verdict } \\
\hline 1 & 6.96 & 1538.1 & 0.86 & 242.6 & 0.61 & 0.52 & Not Good & $\checkmark$ \\
\hline 2 & 16.59 & 2440.3 & 0.81 & 165.1 & 0.97 & 0.80 & Good & $x$ \\
\hline 3 & 7.79 & 1602.9 & 0.97 & 230.8 & 0.96 & 0.92 & Good & $\checkmark$ \\
\hline 4 & 6.43 & 1498.9 & 0.95 & 251.7 & 0.91 & 0.87 & Good & $\checkmark$ \\
\hline \multicolumn{9}{|c|}{ Choice \#3: $\quad$ SIMC with $\tau_{c}=\min \left\{\hat{\tau}_{O L}, \hat{\tau}_{C L}\right\}$} \\
\hline Case \# & $\tau_{C L}$ & $I A E_{0}$ & $\eta_{I A E}$ & $T V_{0}$ & $\eta_{T V}$ & $\eta$ & Verdict & \\
\hline 1 & 7.21 & 1760.8 & 0.75 & 256.9 & 0.64 & 0.48 & Not Good & $\checkmark$ \\
\hline 2 & 16.31 & 2404.6 & 0.81 & 165.2 & 0.97 & 0.78 & (Fairly) Good & $x$ \\
\hline 3 & 7.68 & 1796.5 & 0.87 & 248.6 & 0.98 & 0.85 & Good & $\checkmark$ \\
\hline 4 & 6.46 & 1695.7 & 0.84 & 271.6 & 0.98 & 0.82 & Good & $\checkmark$ \\
\hline
\end{tabular}

values of $\tau_{c}=\hat{\theta}, I A E_{A c t}$, and $T V_{A c t}$ are omitted, being exactly the same of Table 3. It can be observed that values of $I A E_{0}$ and $T V_{0}$ obtained from 26 and 25 are not so far from values obtained with model-based approach. Relative errors of about $15 \%$ are obtained for both indices. Values of performance indices $\left(\eta_{I A E}, \eta_{T V}\right)$ are altered, but values of global index $\eta$ are close, apart for the case \#3. Finally, verdicts on controller tuning are pretty the same.

Note that better performance assessment of case \#3 can be obtained by increasing the value of $M$, that is, augmenting the number of individual ramps, so that errors on lower limits $\left(I A E_{0}, T V_{0}\right)$ are reduced. Such results are not shown in the sake of brevity. Overall, this analysis confirms the validity of proposed approximating equations for lower limits of performance indices, when general-type reference is slow-varying.

Comparing different tuning settings of the reference response. In this section, performance assessment for three different tuning settings of the reference response are analyzed. SIMC and IMC tuning rules, and different choices of $\tau_{c}$ are tested. All results are shown in Table 5 Note that the system in 33 is now simulated with different realizations of the same noise. Therefore, values of $I A E_{A c t}$ and $T V_{A c t}$ are omitted being close to the values of Table 3 .

In details, with the choice \#1, reference PI-type controller $\hat{C}_{r}$ is tuned according to IMC rules and $\tau_{c}=\hat{\theta}$. Note that $\hat{C}_{r}$ has about the same parameters, which are now $K_{c}^{r} \simeq 2.38$ and $\tau_{I}^{r}=$ $\hat{\tau} \simeq 100$, and lower limits of performance indices $\left(I A E_{0}, T V_{0}\right)$ are practically constant. Performance indices are close to values of Table 3, and verdicts are always correct.

With choice \#2, $\hat{C}_{r}$ is tuned according to IMC rules, but $\tau_{c}$ equals the minimum between current time constant, identified in open-loop and in closed-loop: $\tau_{c}=\min \left\{\hat{\tau}_{O L}, \hat{\tau}_{C L}\right\}$. Results of performance assessment are now altered and degraded. Case \#2 becomes a false positive, since aggressive tuning is wrongly assessed as good. Note that, since different values of $\tau_{C L}$ are identified each time, the parameters of reference controller $\left(K_{c}^{r}, \tau_{I}^{r}\right)$ and lower limits of performance indices $\left(I A E_{0}, T V_{0}\right)$ may vary between cases. Note also that, as said before, a fair choice should be $\tau_{c} \in\left[\hat{\theta}, \hat{\tau}_{O L}\right]$, while here two times (for the cases \#1 and \#4) occurs that $\tau_{c}<\hat{\theta}$. Therefore, this choice of tuning settings cannot be considered reliable and effective.

Similar observations apply to choice \#3, in which $\hat{C}_{r}$ is tuned according to SIMC rules and $\tau_{c}=\min \left\{\hat{\tau}_{O L}, \hat{\tau}_{C L}\right\}$.

General remarks. It has to be noted that the presented casestudy is just an example of a wide simulation analysis. The proposed method of performance assessment proves reliable also by using different linear process dynamics (lag/delay dominant), other noise levels, and different trajectories of set-point. Details are omitted for the sake of space.

It is also worth reminding that since valve position $v(t)$ is supposed unknown in the identification stage, an eventual nonlinearity in $V$ makes valve input and output significantly different: $u(t) \neq v(t)$. Therefore, significant errors in process identification would be present: $\hat{P}(t) \neq P(t)$. If a pure linear model is used to identify the whole Hammerstein system (valve plus process), a strong model-mismatch would arise. The valve position could be estimated and stiction amount could be quantified through one of the many methods presented in the literature, 117 which also enables a correct identification of process dynamics.

\section{Application on pilot plant}

In this section some examples of the analysis carried out on a pilot plant are presented. Several sets of data have been collected to confirm the results previously obtained in simulation.

\subsection{Generalities}

The tested control valve - V2 of Figure 6- is a rotative valve with a butterfly shutter. The actuator is of electric type of Rotork $^{\circledR}$ class $C V A$, type $C V Q-90^{\circ}-1200$ (see Figure 7). The controlled variable (PV) is the flow rate of water (expressed in $l / s$ ) through the valve; the control action (OP) is the output signal (0-100\%) of a PI-type controller. The actual position of the valve stem (MV) is measured and controlled internally through the smart electric actuator $A$ with a resolution equal to $0.1 \%$; the sampling period is 1 second (see Figure 8). Being a flow control loop, the process dynamics $P$ substantially coincides with the valve dynamics $V$, conceptually located downstream of actuator $A$.

It has also to be noted that the presented assessment method is totally independent from the type of actuator; therefore, it is equally valid for pneumatic and electric control valves. In general, the availability of MV signal allows one to identify 


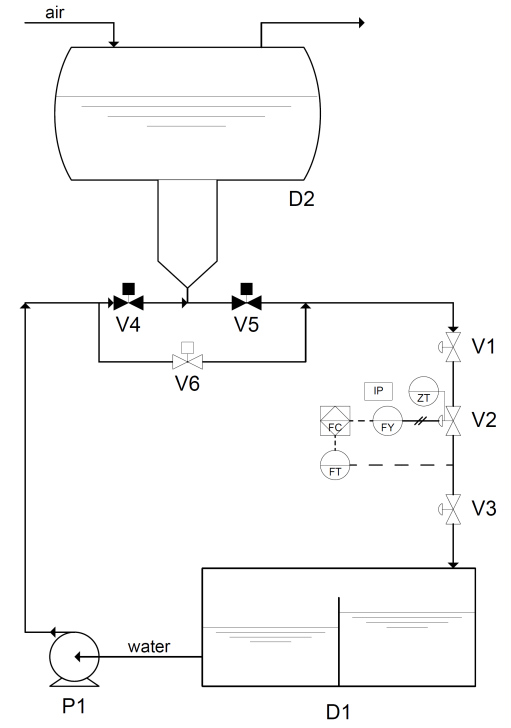

Figure 6: Simplified diagram of the pilot plant.

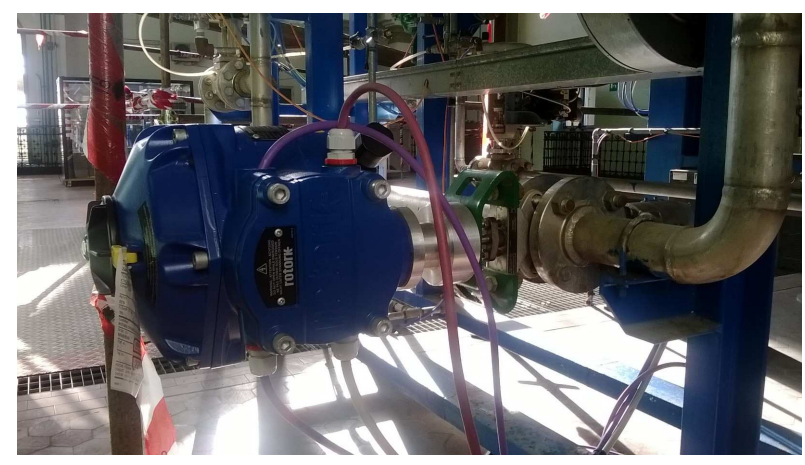

Figure 7: Valve and actuator on-line.

process dynamics directly between $\mathrm{MV}$ and $\mathrm{PV}$, that is, $v(t)$ and $y(t)$. Otherwise, as shown in previous simulations of Section 3 . the method is based on controller output OP, which corresponds to valve position request $u(t)$, and on PV.

Preliminarily to closed-loop tests, some data with controller set in manual have been collected. The valve input is manually imposed to see how valve responds and to register its actual position. These tests are used to verify the absence of any kind of malfunction in the actuator and valve, and to ensure the correct application of the method. Top panel of Figure 9 shows the ramp trends imposed on OP, oscillating from 0 to $100 \%$ of operating range, the actual valve position (MV) and the corresponding flow rate (PV). In addition, bottom panels of Figure 9 show the valve signature on $\mathrm{PV}(\mathrm{OP})$ and $\mathrm{MV}(\mathrm{OP})$ diagrams.

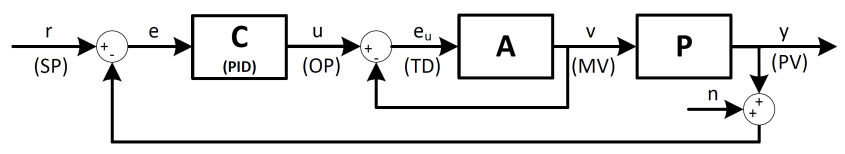

Figure 8: Scheme of control loop with "smart" actuator of electric type.
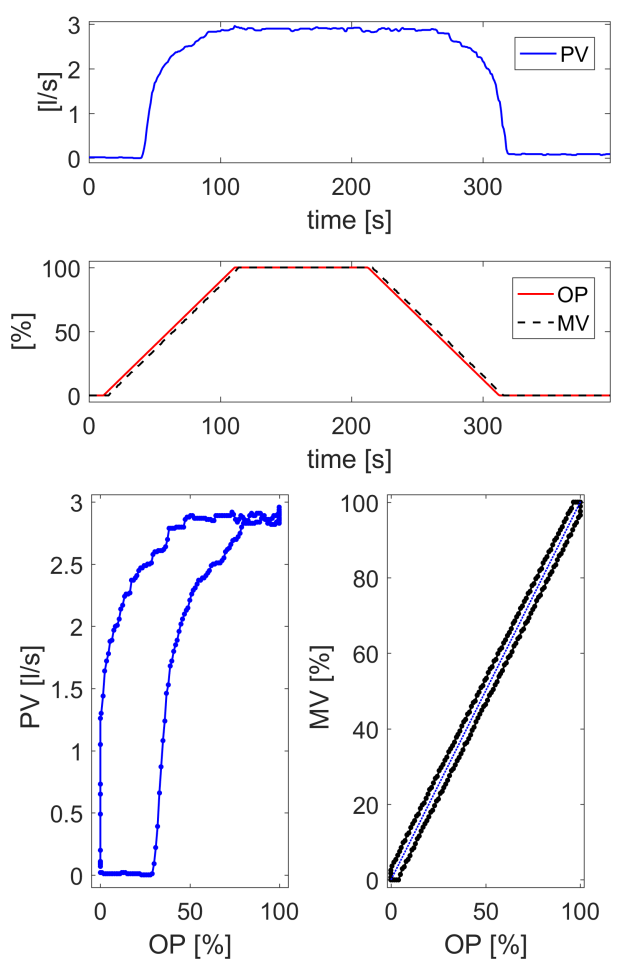

Figure 9: Open-loop test on electric actuator of the pilot plant.

Table 6: Operative conditions for tests on pilot plant.

\begin{tabular}{|c|c|c|c|}
\hline \multirow{2}{*}{ Test \# } & \multicolumn{2}{|c|}{ inverter pump P1 } & Tank D2 \\
\hline & control type & velocity & $\mathrm{LC}$ \\
\hline $0,1,2,3,4$ & manual & constant: $30 \mathrm{~Hz}$ & completed excluded \\
\hline
\end{tabular}

A perfectly linear relationship between position demand and actual position, throughout all the operating range, can be observed. Therefore, malfunctions (non-linearity, as friction) in the actuator can be certainly excluded, which otherwise could have altered results of loop performance analysis.

Four closed-loop tests were carried out under the same operating conditions for the inverter of pump P1 and for the piezometric tank D2, as summarized in Table 6 Other data sets have been collected by operating pump P1 with variable velocity to control level of tank D2. These results are not presented for the sake of brevity. It must be noted that different conditions correspond to different numerical values for optimal (and acceptable) tuning parameters of the controller. In the following paragraphs, details for tests of Table 6 are reported.

\subsection{Preliminary test}

Test 0 has a preliminary nature, since it served to evaluate a suitable range of values for PI controller parameters. Figure 10 shows time trends of controlled variable (PV) for a series of step variations of the reference (SP), and time trends of corresponding control action (OP) and valve position (MV). Table 7 lists the tested values of tuning parameters and corresponding reference changes. 

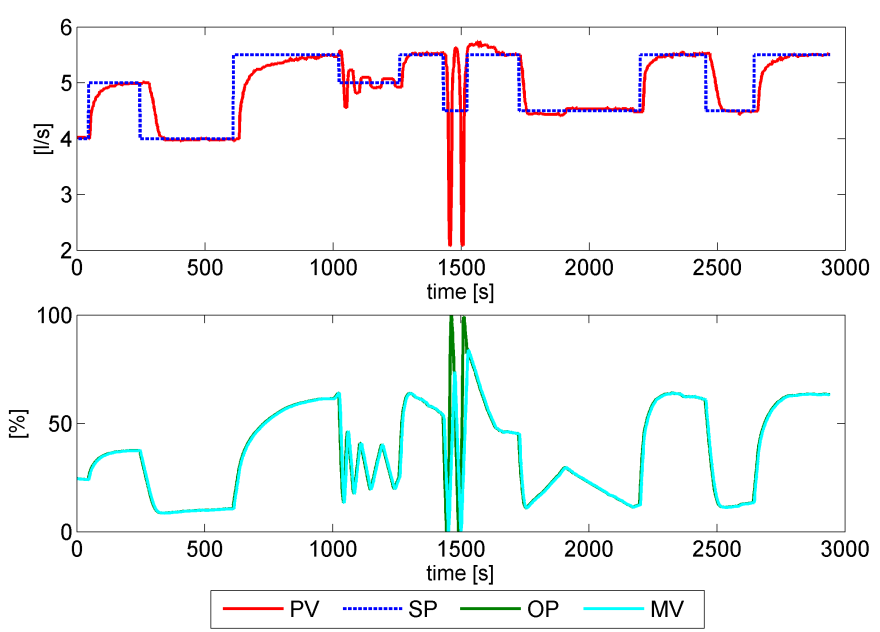

Figure 10: Preliminary test: time trends for set-point changes.

Table 7: Preliminary test: tuning parameters, values of SP and control intervals.

\begin{tabular}{|c|c|c|c|c|c|}
\hline Try & $K_{c}$ & $T_{i}[s]$ & $S P[l / s]$ & time intervals $[s]$ & tuning typologies \\
\hline$\# 1$ & 1 & 2 & $\begin{array}{l}4.0 \rightarrow 5.0 \\
5.0 \rightarrow 4.0 \\
4.0 \rightarrow 5.5\end{array}$ & {$[0 \div 990]$} & rather slow \\
\hline$\overline{\# 2}$ & 6 & 1 & $5.5 \rightarrow 5.0$ & {$[990 \div 1250]$} & very aggressive \\
\hline \#3 & 4 & 1 & $\begin{array}{l}5.0 \rightarrow 5.5 \\
5.5 \rightarrow 4.5\end{array}$ & {$[1250 \div 1520]$} & $\begin{array}{l}\text { very aggressive / } \\
\text { unstable }\end{array}$ \\
\hline$\# 4$ & 4 & 2 & $\begin{array}{l}.5 \rightarrow 5.5 \\
5.5 \rightarrow 4.5 \\
4.5 \rightarrow 5.5\end{array}$ & {$[1520 \div 2440]$} & slightly aggressive \\
\hline$\# 5$ & 2.5 & 2 & $\begin{array}{l}5.5 \rightarrow 4.5 \\
4.5 \rightarrow 5.5\end{array}$ & {$[2440 \div \infty]$} & good \\
\hline
\end{tabular}

It can be observed that the couple $K_{c}=1$ and $T_{i}=2$ (try \#1) represents a rather slow tuning, as it generates over-damped responses with high rise times. In contrast, the pairs of parameters $K_{c}=6 \div 4$ and $T_{i}=1$ (tries \# 2 and \#3) correspond to very aggressive tuning, with obvious fluctuations of all variables. For a value of $S P=4.5 \mathrm{l} / \mathrm{s}$, time trends are even unstable. Furthermore, the pair $K_{c}=4$ and $T_{i}=2$ (try \#4) is a slightly aggressive tuning, because it originates some oscillations on OP in the closing direction of valve. Finally, the pair $K_{c}=2.5$ and $T_{i}=2$ (try \#5) can be considered a good tuning, with the absence of overshoot and no oscillations in both directions of valve input.

\subsection{Validation tests}

The other four experimental tests of Table 6 are used to confirm the validity of index $\eta$ in the evaluation of performance of PID-type control loops. For each of these tests, the set-point is
Table 8: Validation tests: typologies of tuning and corresponding parameters.

\begin{tabular}{llll}
\hline & tuning typologies & $K_{c}$ & $T_{i}$ \\
\hline Test 1 & good & 2.5 & 2 \\
Test 2 & rather sluggish & 1.0 & 2 \\
Test 3 & slightly aggressive & 4.0 & 2 \\
Test 4 & aggressive & 6.0 & 2 \\
\hline
\end{tabular}
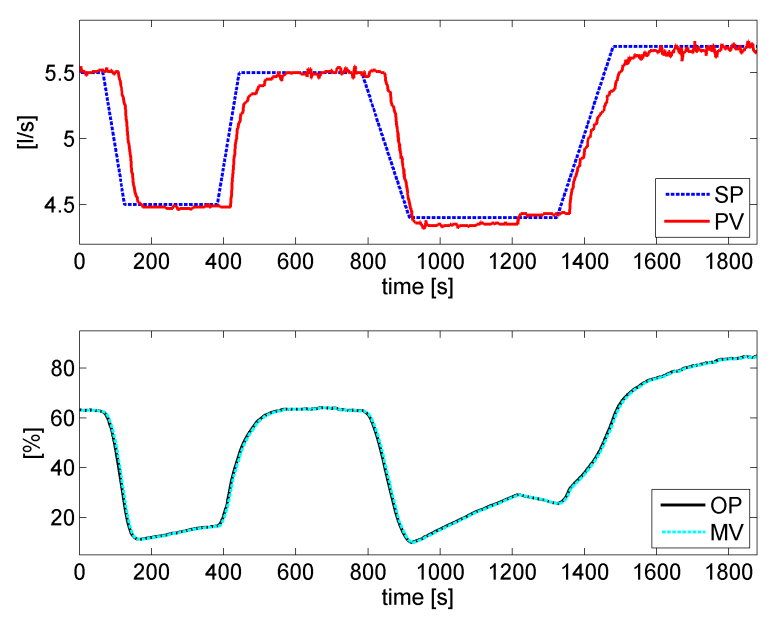

Figure 11: Validation test 1: good tuning.

variable in time as a series of $M=4$ ramps:

$$
r(t)=\left\{\begin{array}{lll}
5.5 & \text { for } \quad 0 \leq t<t_{1} \\
-\frac{1}{60} t+q_{1} & \text { for } t_{1} \leq t<t_{2} \\
4.5 & \text { for } t_{2} \leq t<t_{3} \\
\frac{1}{60} t+q_{2} & \text { for } t_{3} \leq t<t_{4} \\
5.5 & \text { for } t_{4} \leq t<t_{5} \\
-\frac{1}{120} t+q_{3} & \text { for } t_{5} \leq t<t_{6} \\
4.4 & \text { for } t_{6} \leq t<t_{7} \\
\frac{1}{120} t+q_{4} & \text { for } t_{7} \leq t<t_{8} \\
5.7 & \text { for } t_{8} \leq t<t_{\text {fin }}
\end{array}\right.
$$

where $A=[-1,1,-1.1,1.3]$ is the vector of single net amplitudes expressed in $[l / s]$; while $k=$ $[-1 / 60,1 / 60,-1 / 120,1 / 120]$ contains the single slopes in $\left[l / s^{2}\right]$. Finally, $t_{1} \div t_{8}$ and $q_{1} \div q_{4}$, slightly different values for each test, indicate, respectively, time intervals and generic instants, corresponding to intercepts of the ramps. On the basis of indications of the preliminary test (Figure 10), different values of controller parameters are used, as shown in Table 8

The time trend of flow rate in response to variations of reference, the corresponding control action and the valve position are shown in Figure 11, 12, 13, and 14 for different validation tests. Afterwards, on the basis of MV and PV signals, each time a FOPTD model $\hat{P}$ for the process dynamics is identified. Furthermore, on the basis of OP and MV signals, another FOPTD model $\hat{A}$ for actuator dynamics is identified. Results are summarized in Table 9. Note that all the identified models are - correctly - fairly consistent. 

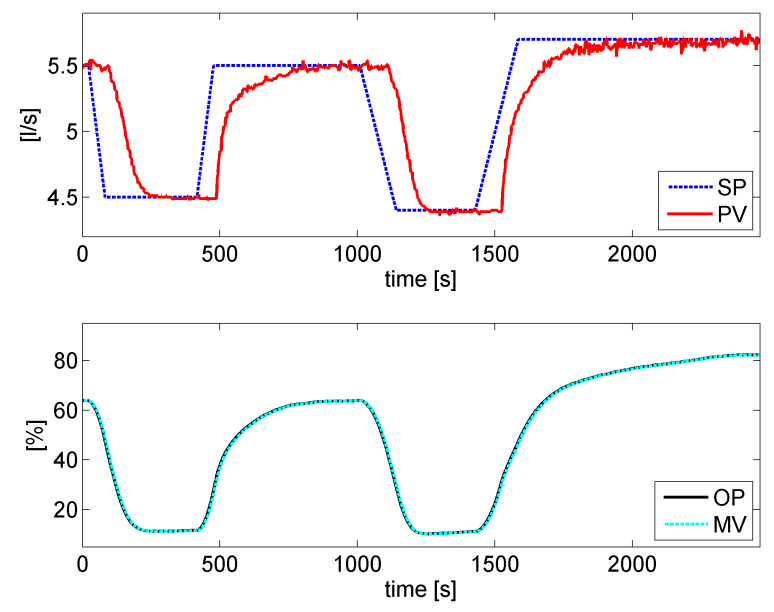

Figure 12: Validation test 2: rather sluggish tuning.
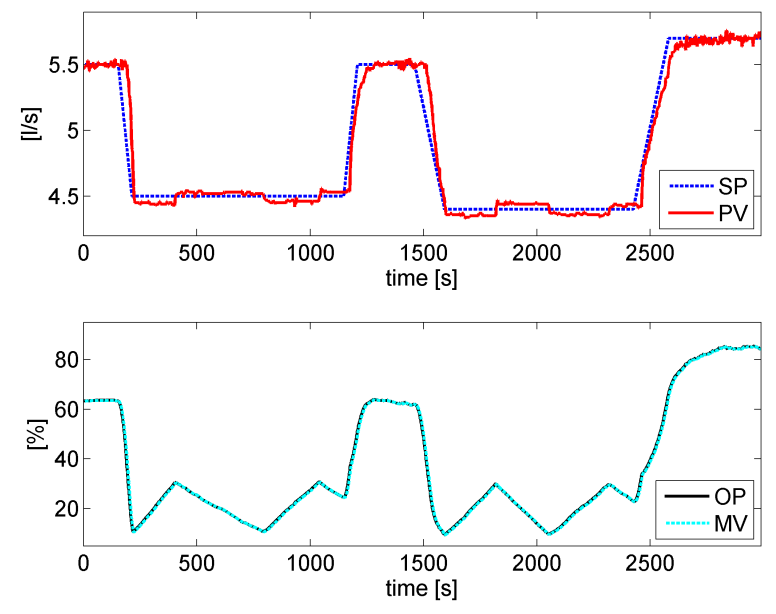

Figure 13: Validation test 3: slightly aggressive tuning.

Then, for each test, on the basis of SP and PV data, the current time constant in closed-loop is estimated $\hat{\tau}_{C L}$, by employing another FOPTD model, as in (10). Note that the reference SIMC-based controller has a PI algorithm, tuned according to (8), since also the global system - valve plus actuator $\left(\hat{P}_{G} \simeq \hat{A} \cdot \hat{P}\right)$ - can be well approximated by a FOPTD model, being the actuator much faster than the process $\left(\hat{\tau}_{P} \approx 100 \hat{\tau}_{A}\right)$, and also being $\hat{\tau}_{A}<\hat{\theta}_{A} \cdot[1]$ Note also that a global time-delay is considered: $\hat{\theta}=\hat{\theta}_{P}+\hat{\theta}_{A}$.

As value for the time constant $\tau_{c}$ of the reference response, the minimum between current time constant, identified in openloop and in closed-loop, is considered: $\tau_{c}=\min \left\{\hat{\tau}_{O L}, \hat{\tau}_{C L}\right\}$, where $\hat{\tau}_{O L}=\hat{\tau}_{P_{G}}$. Note that the choice $\tau=\hat{\theta}$ has to be excluded, since, being $\hat{\theta}$ very small, a too aggressive reference response would be obtained, and results of performance assessment would be negatively affected.

Lower limits of performance indices $\left(I A E_{0}, T V_{0}\right)$ are evaluated with 26) and 25, specially designed for series of ramps. Registered signals of PV and OP are used to compute actual values of indices $\left(I A E_{A c t}, T V_{A c t}\right)$, since the noise level is not
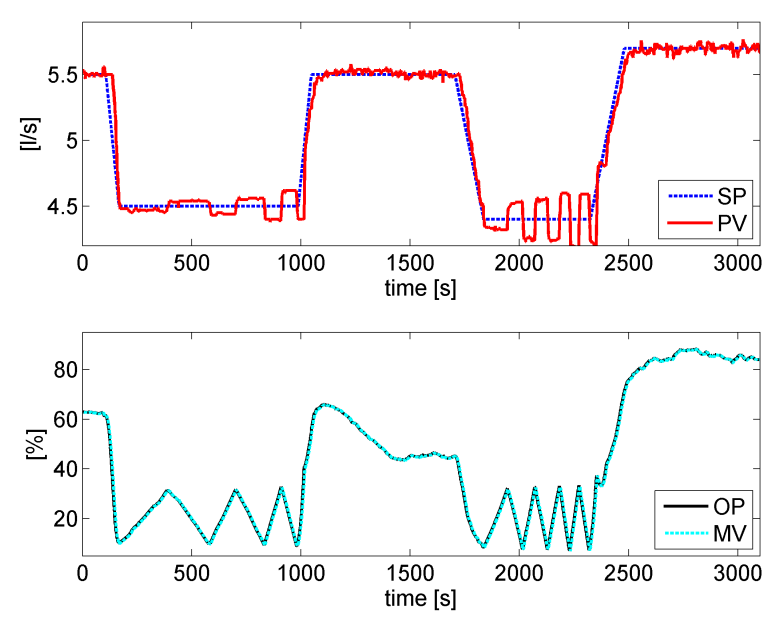

Figure 14: Validation test 4: aggressive tuning.

Table 9: Validation tests. Identified models and parameters.

\begin{tabular}{llcl}
\hline & $\hat{P}$ & $\hat{A}$ & $\hat{P}_{G}$ \\
\hline Test 1 & $\frac{0.023}{62.29 s+1} e^{-0 s}$ & $\frac{1.0000}{0.6070 s+1} e^{-2 s}$ & $\frac{0.023}{62.29 s+1} e^{-2 s}$ \\
Test 2 & $\frac{0.019}{49.44 s+1} e^{-0 s}$ & $\frac{0.9999}{0.584 s+1} e^{-2 s}$ & $\frac{0.019}{49.44 s+1} e^{-2 s}$ \\
Test 3 & $\frac{0.023}{66.23 s+1} e^{-0 s}$ & $\frac{0.9999}{0.635 s+1} e^{-2 s}$ & $\frac{0.023}{66.23 s+1} e^{-2 s}$ \\
Test 4 & $\frac{0.023}{80.66 s+1} e^{-0 s}$ & $\frac{0.9998}{0.597 s+1} e^{-2 s}$ & $\frac{0.023}{80.66 s+1} e^{-2 s}$ \\
\hline
\end{tabular}

excessive and full model-based approach proved not feasible. Note that the closed-loop transfer functions (27) here gives inconsistent time trends. Finally, performance indices related to IAE and TV, and the overall index $\eta$ are computed. All these values are presented in Table 10 . Some brief comments follow.

It can be observed that test 1 , characterized by a good tuning, is actually evaluated as acceptable by the method. The performance index is in fact greater than the threshold value $(\eta=0.81)$; that is, the controller has acceptable performance, which are comparable - although a little inferior - with respect to those theoretically obtainable with the reference SIMC controller. Test 2, expression of a rather sluggish tuning, is actually rated as characterized by unacceptable performance: $\eta=0.47$. In this case, the index $\eta_{I A E}$ is definitely too low, since the actual value for absolute integral error is larger than the reference: $I A E_{A c t}>I A E_{0}$. Also test 3 , expression of a slightly aggressive tuning, is not acceptable: $\eta=0.66$. Once again, index $\eta_{I A E}$ is too low, since $I A E_{A c t}>I A E_{0}$. Finally, test 4, where the controller has a very aggressive tuning, is recognized as clearly

Table 10: Validation tests. Results of loop performance evaluation.

\begin{tabular}{lllllllllll}
\hline & $\tau_{C L}$ & $\tau_{c}$ & $I A E_{0}$ & $I A E_{A c t}$ & $\eta_{I A E}$ & $T V_{0}$ & $T V_{A c t}$ & $\eta_{T V}$ & $\eta$ & $F_{P V}[\%]$ \\
\hline Test 1 & 38.3 & 38.3 & 177.3 & 193.3 & 0.92 & 279.5 & 247.8 & 0.89 & 0.81 & 70.2 \\
Test 2 & 110.0 & 49.4 & 226.3 & 465.6 & 0.49 & 228.3 & 236.6 & 0.97 & 0.47 & 78.3 \\
Test 3 & 25.1 & 25.1 & 119.1 & 177.4 & 0.67 & 358.6 & 367.5 & 0.98 & 0.66 & 74.0 \\
Test 4 & 27.1 & 27.1 & 128.0 & 206.7 & 0.62 & 401.4 & 660.2 & 0.61 & 0.38 & 71.8 \\
\hline
\end{tabular}



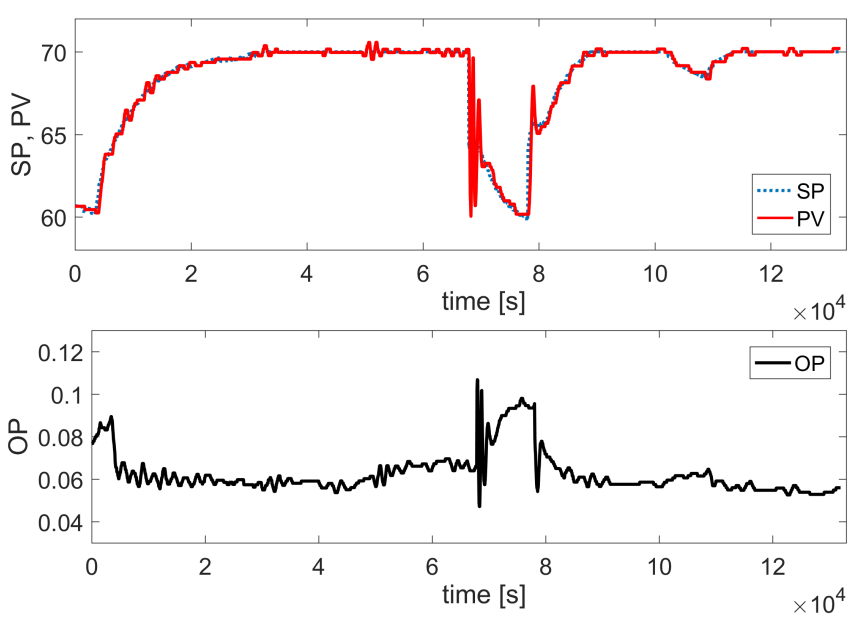

Figure 15: Industrial loop BAS8 with variable set-point.

unacceptable: $\eta=0.38$. Both performance indices are in fact quite low: $\eta_{I A E}=0.62, \eta_{T V}=0.61$, being $I A E_{A c t}>I A E_{0}$, and also $T V_{A c t}>T V_{0}$. Finally, it is to be noted that fitting index $F_{P V}$ is always quite high $(>70 \%)$, close to threshold value, and this gives an indication of validity of process identification, and reliability of performance assessment.

Overall, it can be noticed that, through the proposed refined methodology, it is always possible to have a correct assessment of the performance of control loop and issue a reliable judgment on the validity of controller tuning. Therefore, the initial results obtained in simulation have been confirmed by tests carried out on pilot plant facility.

\section{Industrial data}

In this section, the effectiveness of the performance assessment method is further tested on some industrial data sets with variable reference.

Loop \#1. This first example (BAS8) is taken from a benchmark for stiction detection methods. [9] Data are from the inner loop in a cascade control strategy of a room temperature, and the reference reveals a slow variation. A local portion of 2201 samples $(8300 \div 10500)$, extracted from the whole data set, is assessed and reported in Figure 15. Data do not show any significant oscillation, controller tuning is considered acceptable, and valve stiction and other nonlinearities can be excluded with certainty.

Loop \#2. The second example is a pressure control loop (PC2) from an Italian combined cycle power plant, which is a slave controller in a multi-loop configuration. This loop is subject to frequent set-point changes, due to variable operating conditions which the power plant is forced to because of the interaction with the neighboring renewable energy stations. The controller is known to have acceptable performance and no particular tuning problem is reported from the plant operators. In addition, process nonlinearities can be safely excluded. A data set of 7201 samples is assessed and reported in Figure 16. The general-type set-point reveals a slow variation, which can
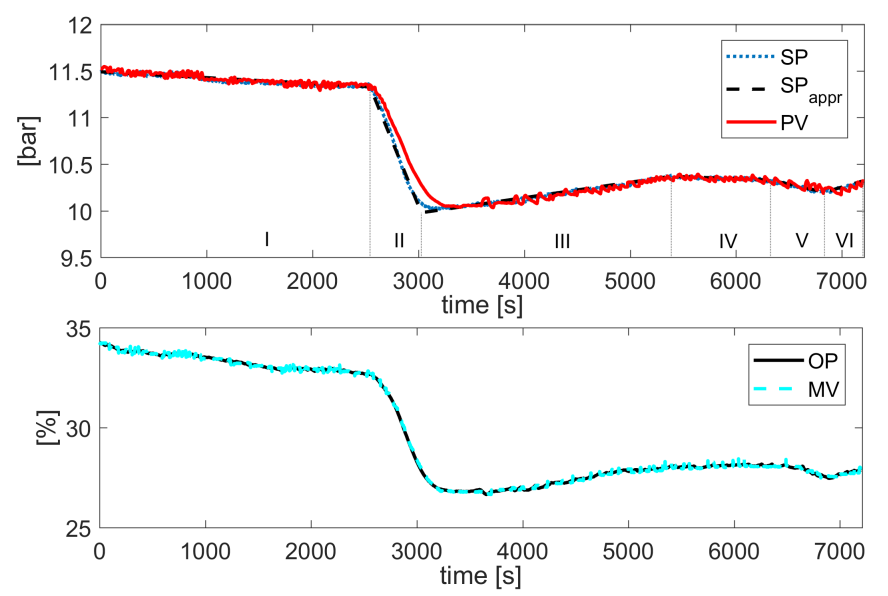

Figure 16: Industrial loop $P C 2$ with variable set-point and series of ramps $\left(S P_{a p p r}\right)$ used as an approximation.

be well approximated by a series of six main changes as ramps $\left(S P_{\text {appr }}\right)$. Lower limits of performance indices $\left(I A E_{0}, T V_{0}\right)$ are here evaluated with 26 and 25 with $M=6$. The controlled variable shows a slightly sluggish response only for the second ramp, the steepest, which occurs about between the 2500th and the 3050th sample. MV and OP signals are almost coincident, so that the absence of malfunctions and nonlinearities in the valve can be inferred.

Loop \#3. The third example is a flow control loop (FC3) from an Italian ethylene plant, with PI control and variable set-point, being the slave loop of a temperature cascade control. ${ }^{[19]}$ This loop is a clear case of incorrect tuning, since $P C U$, a wellestablished software for loop monitoring and assessment, 20$]$ on the basis of 12 acquisitions collected in 24 consecutive hours, emits 10 verdicts of unacceptable performance, with bad controller tuning as the source of malfunction. One of these acquisitions, composed of 7201 samples, is analyzed and presented in Figure 17. Time trends show actually a shifted response with respect to a slow-varying reference, while valve stiction and other nonlinearities are excluded with certainty. Note that, in addition to registered data, Figure 17 shows the estimate of controlled variable $\hat{y}(t)$ and of control action $\hat{u}(t)$, on the basis of the identified process model $\hat{P}(s)$ and the transfer functions in closed-loop mode 27 Reference time trends $\left(\hat{y}_{r}(t), \hat{u}_{r}(t)\right)$ obtained with SIMC-based controller by using (28) are also reported.

Loop \#4. The fourth example (CHEM19), 9 is a flow control loop of an Italian refinery, which is the inner loop in a cascade configuration. The whole data set of 721 samples is assessed and reported in Figure 18 The reference reveals a slow and regular variation, close to a sinusoidal wave, while the process variable shows a square shape, which is a typical indication of static friction in the control valve. Six out of eight detection techniques actually assess the presence of valve stiction. [9] Therefore, the process has a strong nonlinear dynamics and the proposed method is likely to give unreliable results. 

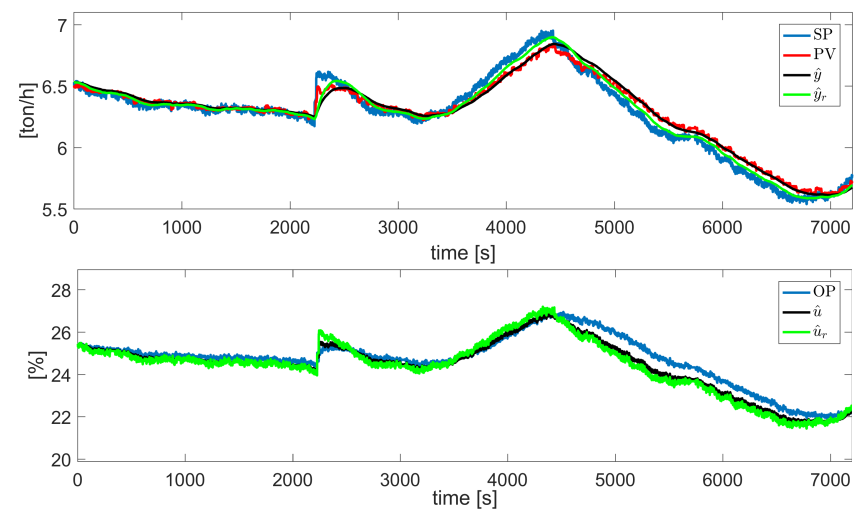

Figure 17: Industrial loop FC3 with variable set-point.
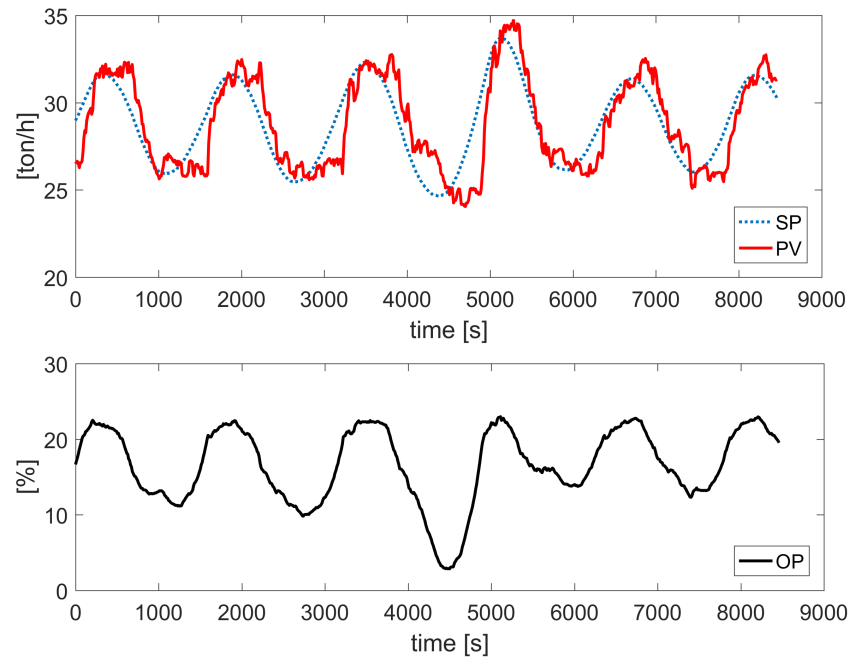

Figure 18: Industrial loop CHEM19 with oscillating set-point and sticky valve.

For each industrial data set, on the basis of control action OP and controlled variable PV signals, a FOPTD model $\hat{P}$ for the process dynamics is identified. The four models are shown in Table 11. Then, on the basis of SP and PV signals, the current time constant in closed-loop $\tau_{C L}$ is estimated, by employing another FOPTD model. As the time constant $\tau_{c}$ of the reference response in closed-loop, the minimum between current time constant, identified in open-loop and in closed-loop, is considered: $\tau_{c}=\min \left\{\hat{\tau}_{O L}, \hat{\tau}_{C L}\right\}$. Then, performance indices for IAE and TV, and global index $\eta$ are computed, as shown in Table 12. Note that for all four control loops, reference variation is of general-type, but definitely slowly varying, so that, approximations committed in 20, and 23) are not too large.

It can be observed that loop $B A S 8$, which shows evidence of good performance, is actually evaluated as very good by the method. The performance index is in fact close to its maximum: $\eta=0.95$. Loop $P C 2$, known to have acceptable perfor-

Table 11: Industrial data: identified FOPTD models.

\begin{tabular}{ccccc}
\hline & BAS8 & PC2 & FC3 & CHEM19 \\
\hline$\hat{P}$ & $\frac{-342.66}{1153.7 s+1} e^{-60 s}$ & $\frac{0.2035}{81.74 s+1} e^{-3 s}$ & $\frac{0.2513}{100.7 s+1} e^{-0 s}$ & $\frac{0.6305}{174.5 s+1} e^{-0 s}$ \\
\hline
\end{tabular}

Table 12: Industrial data. Results of loop performance evaluation.

\begin{tabular}{lllllllllll}
\hline & $\tau_{C L}$ & $\tau_{c}$ & $I A E_{0}$ & $I A E_{A c t}$ & $\eta_{I A E}$ & $T V_{0}$ & $T V_{A c t}$ & $\eta_{T V}$ & $\eta$ & $F_{P V}[\%]$ \\
\hline BAS8 & 384.6 & 384.6 & 15494 & 15645 & 0.99 & 0.409 & 0.428 & 0.96 & 0.95 & 77.18 \\
PC2 & 89.8 & 81.7 & 188.8 & 258.8 & 0.73 & 24.07 & 21.52 & 0.89 & 0.65 & 93.12 \\
FC3 & 79.7 & 79.7 & 257.1 & 453.5 & 0.57 & 298.2 & 197.6 & 0.66 & 0.38 & 90.51 \\
CHEM19 & 130.0 & 130.0 & 7792 & 10718 & 0.73 & 121.0 & 151.1 & 0.80 & 0.58 & 62.70
\end{tabular}

mance, is actually assessed as pretty fair, but a bit improvable. The global index is indeed under threshold value $(\eta=0.65)$, since $I A E_{A c t}>I A E_{0}$. By using a controller based on SIMC tuning rules, it would have been possible to reduce control error in tracking the steep ramp change without increasing too much the total variation of control action. Loop FC3, known to have a sluggish controller, is correctly assessed with clear unacceptable performance: $\eta=0.38$. Both indices are in fact quite low $\left(\eta_{I A E}=0.57, \eta_{T V}=0.66\right)$, being $I A E_{A c t}>I A E_{0}$ and $T V_{A c t}<T V_{0}$. Finally, loop CHEM19, known to be affected by valve stiction, is assessed as not acceptable: $\eta=0.58$. However, this verdict cannot be considered reliable, since the fitting index on controlled variable is quite low: $F_{P V}=62.7 \%$. For other three loops, $F_{P V}$ is definitely higher, which gives an indication of validity of process identification, and reliability of performance assessment.

Overall, these applications on industrial data confirm good results previously obtained in simulation and then on the pilot plant.

\section{Conclusions}

This paper presented a refined method for performance analysis in the case of reference variations, based on some recent works of literature on this subject. In particular, the index $(\eta)$ for performance assessment takes into account not only the control error, but also the required control action. The study has been extended in order to cover almost every type of set-point changes, which can be represented in terms of a series of small ramp variations. Applications on different typologies of data show the validity of the method. Several simulation examples and experimental validation tests conducted on a pilot plant have been presented. Finally, the method has been successfully applied to routine data obtained from different industrial processes. Note that a preliminary step to exclude the presence of nonlinearities is necessary, since, in order to set reference values for tuning parameters of PID-type controller, the identified process model has to be linear. The relative simplicity of the technique makes it appealing for industrial application in the cases of continuous set-point changes, as encountered nowadays in traditional power plants for operation with transient loads. The results are considered valid and will be included in the last version of the monitoring software system $P C U,[20$ by representing an improvement to its assessment capabilities.

\section{References}

[1] I. Graabak, M. Korpås, Energies 2016, 9, 449.

[2] J. Gostling, Operation, Maintenance and Material Issues 2002, 1, 1. 
[3] S. Mills, Profiles 2011, 1,1 .

[4] B. Huang, S. L. Shah, Performance Assessment of Control Loops: Theory and Applications, 1st edition, Springer-Verlag, London, 1999.

[5] M. Jelali, Control Performance Management in Industrial Automation: Assessment, Diagnosis and Improvement of Control Loop Performance, 1st edition, Springer-Verlag, London, 2013.

[6] O. Arrieta, R. Vilanova, Ind. Eng. Chem. Res. 2012, 51, 2666.

[7] Z. Yu, J. Wang, B. Huang, Z. Bi, J. Proc. Contr. 2011, 21, 1164.

[8] Z. Yu, J. Wang, B. Huang, Z. Bi, Ind. Eng. Chem. Res. 2014, 53, 11050.

[9] M. Jelali, B. Huang, Detection and Diagnosis of Stiction in Control Loops: State of the Art and Advanced Methods, 1st edition, SpringerVerlag, London, 2010.

[10] D. Rivera, M. Morari, S. Skogestad, Ind. Eng. Chem. Process Des. Dev. 1986, $25,252$.

[11] S. Skogestad, J. Proc. Contr. 2003, 13, 291.

[12] S. Björklund, L. Ljung, "A review of time-delay estimation techniques," 42nd IEEE Conference on Decision \& Control, Maui, Hawaii USA, December 2003.

[13] M. Veronesi, A. Visioli, Ind. Eng. Chem. Res. 2009, 48, 2616.

[14] M. Veronesi, A. Visioli, ISA Trans. 2010, 49, 244.

[15] A. Swanda, D. Seborg, "Controller performance assessment based on setpoint data," 6th American Control Conference, San Diego, California USA, June 1999.

[16] J. W. V. Dambros, M. Farenzena, J. O. Trierweiler, Ind. Eng. Chem. Res. 2016, 55, 10316.

[17] R. Bacci di Capaci, C. Scali, G. Pannocchia, J. Proc. Contr. 2016, 46, 11

[18] A. Brambilla, C. Scali, S. Chen, Hydrocarbon Processing 1990, 69, 11.

[19] R. Bacci di Capaci, C. Scali, E. Rossi, F. Gomiero, A. Pagano, Chemical Engineering Transactions 2015, 43, 1369.

[20] C. Scali, M. Farnesi, Annual Reviews in Control 2010, 34, 263. 